\title{
Correction to: Gait Patterns Monitoring Using Instrumented Forearm Crutches
}

\author{
Marien Narváez (ib) and Joan Aranda (1)
}

\section{Correction to: \\ Chapter "Gait Patterns Monitoring Using Instrumented \\ Forearm Crutches" in: K. Miesenberger et al. (Eds.): \\ Computers Helping People with Special Needs, LNCS 12377, https://doi.org/10.1007/978-3-030-58805-2_48}

The original version of this chapter was revised. The chapter was published open access, but this has been reversed and the copyright is now (C) Springer Nature Switzerland AG. 